\title{
Decreasing rates of venous thromboembolism after radical cystectomy: an encouraging trend and more work is still needed
}

\author{
Kristen McAlpine ${ }^{1}$, Luke T. Lavallée ${ }^{1,2}$ \\ ${ }^{1}$ Division of Urology, ${ }^{2}$ The Ottawa Hospital Research Institute, University of Ottawa, Ottawa, ON, Canada \\ Correspondence to: Luke T. Lavallée, MDCM, MSc, FRCSC. The Ottawa Hospital Research Institute, Urologic Oncologist, Division of Urology, The \\ University of Ottawa, General Campus, 501 ch. Smyth Rd. Box/C.P. 222, Ottawa, ON, K1H 8L6, Canada. Email: lulavallee@toh.ca. \\ Provenance: This is a Guest Editorial commissioned by Section Editor Xiao Li (Department of Urology, Jiangsu Cancer Hospital \& Jiangsu Institute \\ of Cancer Research \& Nanjing Medical University Affiliated Cancer Hospital, Nanjing, China). \\ Comment on: Lyon TD, Tollefson MK, Shah PH, et al. Temporal trends in venous thromboembolism after radical cystectomy. Urol Oncol \\ 2018;36:361.e15-361.e21.
}

Submitted Jul 22, 2018. Accepted for publication Jul 26, 2018.

doi: $10.21037 /$ tau.2018.07.15

View this article at: http://dx.doi.org/10.21037/tau.2018.07.15

Venous thromboembolism (VTE) following major cancer surgery is a significant cause of preventable morbidity and mortality. VTE is the leading cause of non-cancer related death after abdominopelvic surgery for cancer, and improvements in risk assessment as well as strategies for prevention and treatment are needed (1).

In this study by Lyon et al. published in May 2018, the temporal trend in VTE rates after radical cystectomy were assessed using the American College of Surgeons' National Surgical Quality Improvement Program (NSQIP) (2). This study reported decreasing rates of VTE after radical cystectomy from $5.1 \%$ in 2011 to $2.8 \%$ in 2016 (2).

The authors also identified independent risk factors for VTE after cystectomy including active malignancy, long operative time, obesity and infection (2). These findings are consistent with prior literature (3). Risk factors for VTE have previously been studied and have been incorporated into risk assessment tools that surgeons may use to risk stratify patients. Unfortunately, some of these tools may be challenging to use due to the multitude of variables they include, or may not sufficiently account for operative factors leading to insufficient risk stratification for some procedures (3).

Preventing VTEs is important, and this study appears to demonstrate that urologists have been successful in reducing VTE rates after cystectomy: the urological procedure historically associated with the highest rate of VTE (3). Prevention of VTEs has been identified as a focus for quality improvement programs in post-operative care. Over recent years, recommendations have focused on extending the duration of thromboprophylaxis after surgery to reduce VTEs that occur after discharge from hospital. Indeed, this study reports that almost half (49\%) of VTEs in patients receiving cystectomy occur after hospital discharge, consistent with previous studies which include other urological procedures $(3,4)$.

The time period examined in this study is of interest because it spans a time when important changes to thromboprophylaxis guideline recommendations were made. In 2012, the American College of Chest Physicians (ACCP) published landmark guidelines on venous thromboprophylaxis following non-orthopedic operations (5). This publication was the best recognized guide for VTE prophylaxis following abdominal and pelvic surgery (including urological surgery) prior to the recently published European Association of Urology (EAU) procedure-specific guidelines (6). It is plausible that some of the improvements in VTE rates observed in this study are attributable to a change in practice patterns and the uptake of extended duration thromboprophylaxis following this publication.

A noteworthy strength of this study is that the results may indicate improvements in the quality of post-cystectomy care internationally. NSQIP has member institutions across the world, making the results more generalizable than most cystectomy series that are derived from a single or a few large academic centers. Because NSQIP does not 
contain granular data for all disease factors or treatments (prophylaxis) received, the study is unable to determine why VTE rates have decreased. Institutions enrolled in NSQIP are often larger and more academic, therefore it is possible hospital or system factors, rather than clinician practice, are contributing to improvements in VTE rates seen in this study. Factors such as peri-operative VTE prophylaxis care pathways and enhanced recovery after surgery (ERAS) protocols now used at many institutions, may reduce VTE rates by promoting earlier ambulation, less infections, and a more rapid resumption of regular activity.

As the authors acknowledge, NSQIP data has several important limitations when used to study VTE. Most importantly, information including the use of venous thromboprophylaxis, neoadjuvant chemotherapy, and oncological factors such as tumor grade and stage, are not included in NSQIP and therefore could not be adjusted for in this study. In addition, while it is highly encouraging to see VTE rates decrease, it would be more informative to have these data paired with bleeding rates at cystectomy in the same population. The benefits of thromboprophylaxis must always be balanced with the possible increased risk of major bleeding and transfusion. Future studies to assess VTE and bleeding rates over time are needed.

This study provides positive news for patients with bladder cancer who have amongst the highest burden of care of all urological patients and we commend the authors for their work. Despite these positive results, there remains much room for improvement in the prevention of VTE following urological surgery. Studies examining the timing, type, duration and effectiveness of medical and mechanical thromboprophylaxis in urology are still needed. Furthermore, tools to improve and facilitate risk stratification applicable to all urological procedures are required.

Cite this article as: McAlpine K, Lavallée LT. Decreasing rates of venous thromboembolism after radical cystectomy: an encouraging trend and more work is still needed. Transl Androl Urol 2018;7(6):982-983. doi: 10.21037/tau.2018.07.15

\section{Acknowledgements}

None.

\section{Footnote}

Conflicts of Interest: LT Lavallee has received research grants from Sanofi and been an ad board participant for Sanofi and Ferring, unrelated to this work. K McAlpine has no conflicts of interest to declare.

\section{References}

1. Agnelli G, Bolis G, Capussotti L, et al. A Clinical OutcomeBased Prospective Study on Venous Thromboembolism After Cancer Surgery. Ann Surg 2006;243:89-95.

2. Lyon TD, Tollefson MK, Shah PH, et al. Temporal trends in venous thromboembolism after radical cystectomy. Urol Oncol 2018;36:361.e15-361.e21.

3. McAlpine K, Breau RH, Mallick R, et al. Current guidelines do not sufficiently discriminate venous thromboembolism risk in urology. Urol Oncol 2017;35:457.e1-e8.

4. Lavallée LT, Schramm D, Witiuk K, et al. Perioperative morbidity associated with radical cystectomy in a multicenter database of community and academic hospitals. PLoS One 2014;9:e111281.

5. Gould MK, Garcia DA, Wren SM, et al. Prevention of VTE in nonorthopedic surgical patients: Antithrombotic Therapy and Prevention of Thrombosis, 9th ed: American College of Chest Physicians Evidence-Based Clinical Practice Guidelines. Chest 2012;141:e227S-77S.

6. Tikkinen KAO, Craigie S, Agarwal A, et al. Procedurespecific Risks of Thrombosis and Bleeding in Urological Non-cancer Surgery: Systematic Review and Metaanalysis. Eur Urol 2018;73:236-41. 